\title{
TEST PROBLEMS FOR VALIDATION OF SPACE CHARGE CODES
}

\author{
Yuri K. Batygin \\ The Institute of Physical and Chemical Research (RIKEN), Saitama, 351-01, Japan
}

\section{Abstract}

Particle-in-cell codes for intense beam dynamics study with space charge include parameters defined by user: number of modeling particles, number of grid points and integration step. Combination of that parameters has to provide the most accurate calculation of beam dynamics during reasonable computing time. Analytical solutions for intense beam dynamics problems with space charge allow comparable runs of codes with controlled accuracy of calculations. In present paper test problems using PIC code BEAMPATH are given.

\section{INTRODUCTION}

Self-consistent solutions for beam distribution function serve as test problems for verification of beam space charge codes. Most of the solutions are obtained for beam with linear space charge forces. Large number of tests are available utilizing $\mathrm{KV}$ equations for beam envelopes. Here we consider drift of uniform beam in free space (Section 2). Problem of beam drift is generalized for non-uniform beam as well (Section 3). Number of tests for both uniform and non-uniform beams are available for beam equilibria with space charge (Section 4). Several tests (drift of uniformly charged ellipsoid, Section 5 and beam bunching, Section 6) include longitudinal space charge forces.

\section{DRIFT OF UNIFORM BEAM IN FREE SPACE}

Spread of round uniform beam with current I, emittance $\ni$, velocity $\beta$ and energy $\gamma$ in free space is described by $\mathrm{KV}$ equation for beam envelope $\mathrm{R}$ [1]:

$$
\frac{\mathrm{d}^{2} \mathrm{R}}{\mathrm{dz}}-\frac{\ni^{2}}{\mathrm{R}^{3}}-\frac{2 \mathrm{I}}{\mathrm{R}(\beta \gamma)^{3} \mathrm{I}_{\mathrm{c}}}=0,
$$

where $I_{c}=4 \pi \varepsilon_{0} \mathrm{mc}^{3} / \mathrm{q}$ is a characteristic value of beam current. For space charge dominated beam $\ni \approx 0$, Eq. (1) has a solution [2]:

$$
\frac{\mathrm{R}}{\mathrm{R}_{\mathrm{o}}}=1+0.25 \mathrm{Z}^{2}-0.017 \mathrm{Z}^{3}, \mathrm{Z}=\frac{\mathrm{Z}}{\mathrm{R}_{\mathrm{o}}} \sqrt{\frac{4 \mathrm{I}}{\mathrm{I}_{\mathrm{c}} \beta^{3} \gamma^{3}}} .
$$

In Fig. 1 results of beam envelope evolution utilizing $10^{4}$ particles on the grid $256 \times 256$ are presented. Deviation from analytical solution (2) is less than $10^{-4}$.

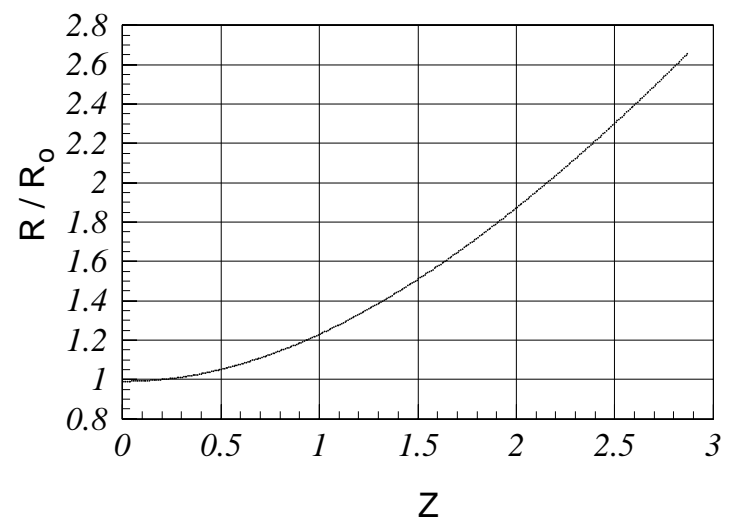

Fig. 1. Drift of uniform beam in free space.

\section{DRIFT OF GAUSSIAN BEAM IN FREE SPACE}

If beam is not uniform, it's density profile as well as beam sizes are changed in drift space. Evolution of Gaussian beam with zero emittance in drift space under self non-linear space charge forces is described by expression [3]:

$$
\rho=\frac{\rho_{o} \exp \left(-2 \xi_{o}^{2}\right)}{a_{o}+a_{1} F+a_{2} F^{2}+a_{3} F^{3}+a_{4} F^{4}+a_{5} F^{5}+a_{6} F^{6}},
$$

where the following notations are used:

$$
\xi_{\mathrm{o}}=\frac{\mathrm{r}_{\mathrm{o}}}{\mathrm{R}_{\mathrm{o}}}, \mathrm{F}=\sqrt{\frac{1-\exp \left(-2 \xi_{\mathrm{o}}^{2}\right)}{\xi_{\mathrm{o}}^{2}}}, \eta=\frac{4 \mathrm{I}}{\mathrm{I}_{\mathrm{c}} \beta^{3} \gamma^{3}} \frac{\mathrm{z}^{2}}{\mathrm{R}_{\mathrm{o}}^{2}},
$$

$a_{o}=1+\eta \exp \left(-2 \xi_{o}^{2}\right), a_{1}=-0.102 \eta^{3 / 2} \exp \left(-2 \xi_{o}^{2}\right)$

$\mathrm{a}_{2}=\frac{1}{4} \eta^{2} \exp \left(-2 \xi_{\mathrm{o}}^{2}\right)$

$\mathrm{a}_{3}=0.017 \eta^{3 / 2}-0.0425 \eta^{5 / 2} \exp \left(-2 \xi_{\mathrm{o}}^{2}\right)$,

$\mathrm{a}_{4}=1.734 \cdot 10^{-3} \eta^{3} \exp \left(-2 \xi_{\mathrm{o}}^{2}\right)-\frac{1}{16} \eta^{2}$

$\mathrm{a}_{5}=0.01275 \eta^{5 / 2}, \quad \mathrm{a}_{6}=-5.78 \cdot 10^{-4} \eta^{3}$.

In Fig. 2 results of evolution of $35 \mathrm{MeV}, 4.7 \mathrm{~A}, \mathrm{D}^{+}$ beam with initial Gaussian distribution and initial radius of $\mathrm{R}_{\mathrm{O}}=1.3 \mathrm{~cm}$ are presented. Simulations with $10^{4}$ particles on the grid 256 × 256 indicate good agreement with theory. 

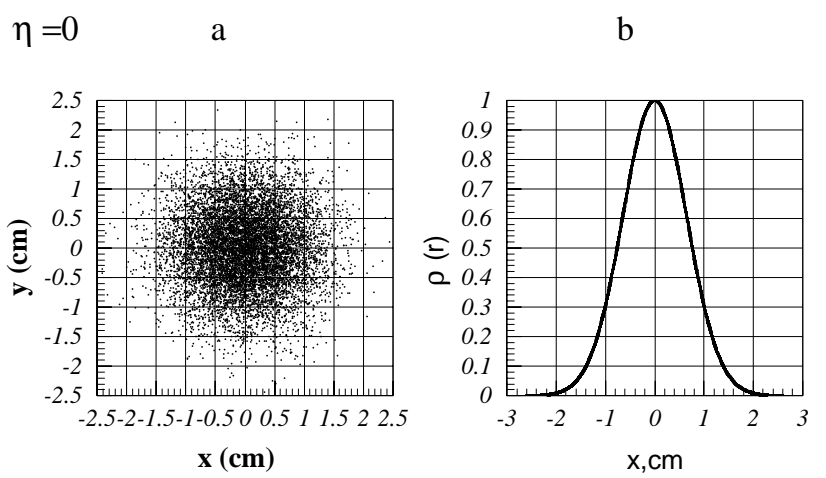

$\eta=3.8$
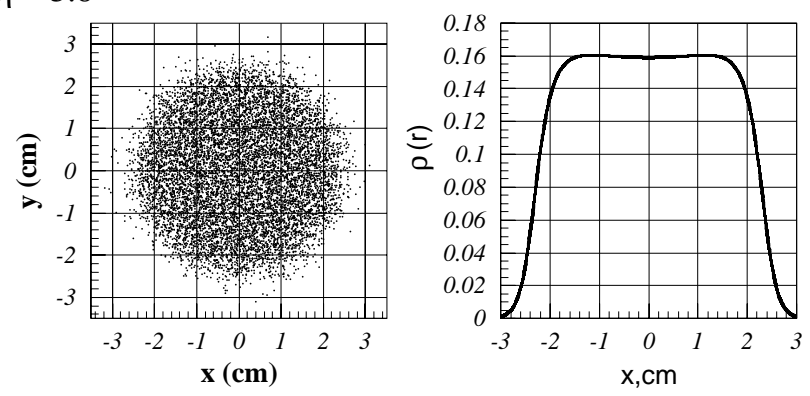

Fig. 2. Redistribution of Gaussian beam in drift space: aPIC simulation, b - calculated from Eq. (3).

\section{BEAM EQUILIBRIUM WITH SPACE CHARGE}

If beam distribution function $\mathrm{f}\left(\mathrm{x}, \mathrm{y}, \mathrm{p}_{\mathrm{x}}, \mathrm{p}_{\mathrm{y}}\right)$ is conserved in time-independent focusing field, beam is in equilibrium with external field. Stationary self-consistent beam distribution function is a solution of VlasovPoisson's equations:

$$
\left\{\begin{array}{l}
\frac{1}{\mathrm{~m} \gamma}\left(\frac{\partial \mathrm{f}}{\partial \mathrm{x}} \mathrm{p}_{\mathrm{x}}+\frac{\partial \mathrm{f}}{\partial \mathrm{y}} \mathrm{p}_{\mathrm{y}}\right)-\mathrm{q}\left(\frac{\partial \mathrm{f}}{\partial \mathrm{p}_{\mathrm{x}}} \frac{\partial \mathrm{U}}{\partial \mathrm{x}}+\frac{\partial \mathrm{f}}{\partial \mathrm{p}_{\mathrm{y}}} \frac{\partial \mathrm{U}}{\partial \mathrm{y}}\right)=0 \\
\frac{1}{\mathrm{r}} \frac{\partial}{\partial \mathrm{r}}\left(\mathrm{r} \frac{\partial \mathrm{U}_{\mathrm{b}}}{\partial \mathrm{r}}\right)=-\frac{\mathrm{q}}{\varepsilon_{\mathrm{o}}} \int_{-\infty}^{\infty} \int_{-\infty}^{\infty} \mathrm{f}\left(\mathrm{x}, \mathrm{p}_{\mathrm{x}}, \mathrm{y}, \mathrm{p}_{\mathrm{y}}\right) \mathrm{dp_{ \textrm {x } }} d \mathrm{p}_{\mathrm{y}}
\end{array}\right.
$$

where $U=U_{b}+\gamma^{-2} U_{e x t}$ is a total potential of the structure, $U_{b}$ is a space charge potential and $U_{\text {ext }}$ is an external focusing potential. General treatment of the problem (5) for arbitrary distribution function was given in [4]. In Table 1 self-consistent solutions for different beam distributions are given.

\section{DRIFT OF UNIFORMLY CHARGED ELLIPSOID IN FREE SPACE}

All mentioned problems dealt with transverse particle motion. Several tests are available including longitudinal space charge forces. Consider uniformly populated spheroid with charge $\mathrm{Q}$ and $\mathrm{a}, \mathrm{b}$, as longitudinal and transverse semi-axes, respectively. Potential of the uniformly populated ellipsoid in free space is given by

$$
\begin{array}{ll}
U=-\frac{\rho}{2 \varepsilon_{o}}\left[\left(\frac{1-M_{z}}{2}\right) r^{2}+M_{z} z^{2}\right], & \rho=\frac{3}{4 \pi} \frac{Q}{b^{2} a} \\
M_{z}=\frac{1-\varepsilon^{2}}{\varepsilon^{2}}\left[\frac{1}{2 \varepsilon} \ln \left(\frac{1+\varepsilon}{1-\varepsilon}\right)-1\right], & \varepsilon=\frac{\sqrt{a^{2}-b^{2}}}{a} .
\end{array}
$$

Space charge forces inside ellipsoid are

$$
\mathrm{E}_{\mathrm{r}}=\frac{3}{8 \pi \varepsilon_{\mathrm{o}}} \frac{\mathrm{Q}}{\mathrm{b}^{2} \mathrm{a}}\left(1-\mathrm{M}_{\mathrm{z}}\right) \mathrm{r}, \quad \mathrm{E}_{\mathrm{z}}=\frac{3}{4 \pi \varepsilon_{\mathrm{o}}} \frac{\mathrm{Q}}{\mathrm{b}^{2} \mathrm{a}} \mathrm{M}_{\mathrm{z}} \mathrm{z}
$$

Due to linear space charge forces, ellipsoid remains uniformly populated in drift space. Equations for evolution of ellipsoid boundaries are

$$
\frac{\mathrm{d}^{2} \mathrm{a}}{\mathrm{dt}^{2}}=\frac{3}{4 \pi} \frac{\mathrm{q}}{\mathrm{m} \varepsilon_{\mathrm{o}}} \frac{\mathrm{Q} \mathrm{M}_{\mathrm{z}}}{\mathrm{b}^{2}}, \quad \frac{\mathrm{d}^{2} \mathrm{~b}}{\mathrm{dt}^{2}}=\frac{3}{8 \pi} \frac{\mathrm{q}}{\mathrm{m} \varepsilon_{\mathrm{o}}} \frac{\mathrm{Q}\left(1-\mathrm{M}_{\mathrm{z}}\right)}{\mathrm{ab}} \text {. }
$$

In Fig. 3. numerical results for ellipsoid with $Q=3$ nK utilizing $2 \cdot 10^{4}$ particles on the grid $128 \times 512$ are presented. Initial ellipsoid with ratio of semi-axes $\mathrm{a} / \mathrm{b}=3$ is transformed into ellipsoid with ratio of $\mathrm{a} / \mathrm{b}=1.55$ (analytical solution $\mathrm{a} / \mathrm{b}=1.62$ ). General treatment of ellipsoid drift in free space including finite value of beam emittance is given in [5].
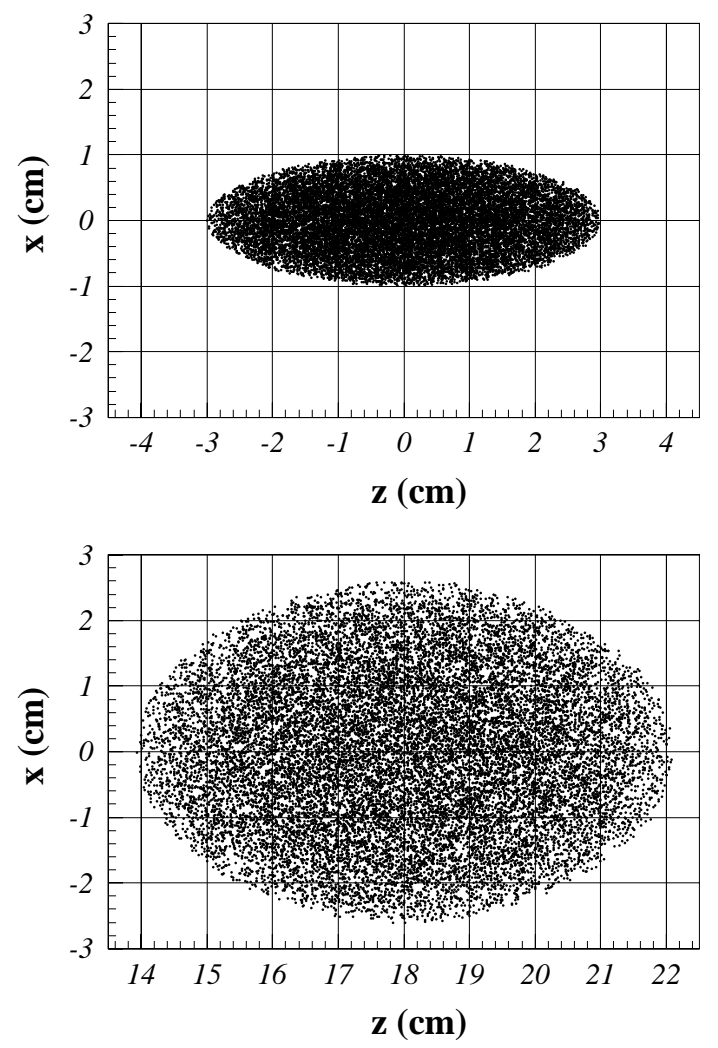

Fig. 3. Drift of uniformly populated ellipsoid in free space. 
Table 1. Self-consistent beam equilibrium with space charge.

Distribution function

KV

Gaussian

$$
f=f_{o} \delta\left(\frac{p_{x}^{2}+p_{y}^{2}}{(\varepsilon / R)^{2}}+\frac{x^{2}+y^{2}}{R^{2}}-H_{o}\right)
$$

$$
f=f_{o} \exp \left(-2 \frac{p_{x}^{2}+p_{y}^{2}}{(\varepsilon / R)^{2}}-2 \frac{x^{2}+y^{2}}{R^{2}}\right)
$$

Water bag $\quad \mathrm{f}=\mathrm{f}_{\mathrm{o}}, \quad \frac{2}{3}\left(\frac{\mathrm{x}^{2}+\mathrm{y}^{2}}{\mathrm{R}^{2}}+\frac{\mathrm{p}_{\mathrm{x}}^{2}+\mathrm{p}_{\mathrm{y}}^{2}}{(\varepsilon / \mathrm{R})^{2}}\right) \leq 1$

Parabolic $\quad f=f_{o}\left(1-\frac{x^{2}+y^{2}}{2 R^{2}}-\frac{p_{x}^{2}+p_{y}^{2}}{2(\varepsilon / R)^{2}}\right)$

Extended Gaussian $\mathrm{f}=\mathrm{f}_{\mathrm{o}} \exp \left(-2 \frac{\mathrm{p}_{\mathrm{x}}^{2}+\mathrm{p}_{\mathrm{y}}^{2}}{(\varepsilon / \mathrm{R})^{2}}-\frac{\left(\mathrm{x}^{2}+\mathrm{y}^{2}\right)^{2}}{\mathrm{R}^{4}}\right)$

$$
\begin{aligned}
& E(r)=-\frac{m c^{2}}{q R} \frac{1}{\gamma}\left(\frac{r}{R}\right)\left[\left(\frac{\varepsilon}{R}\right)^{2}+2 \frac{I}{\beta \gamma I_{c}}\right] \\
& E(r)=-\frac{m c^{2}}{q R \gamma} \frac{r}{R}\left[\frac{\varepsilon^{2}}{R^{2}}+\frac{4 I}{\beta \gamma I_{c}} \frac{\left(1-\exp \left(-2 r^{2} / R^{2}\right)\right.}{2\left(r^{2} / R^{2}\right)}\right]
\end{aligned}
$$$$
E(r)=-\frac{m c^{2} r}{q R^{2} \gamma}\left[\frac{\varepsilon^{2}}{R^{2}}+\frac{8 I}{3 I_{c} \beta \gamma}\left(1-\frac{r^{2}}{3 R^{2}}\right)\right]
$$$$
E(r)=-\frac{m c^{2} r}{q R^{2} \gamma}\left[\frac{\varepsilon^{2}}{R^{2}}+\frac{3 I}{I_{c} \beta \gamma}\left(1-\frac{r^{2}}{2 R^{2}}+\frac{r^{4}}{12 R^{4}}\right)\right]
$$$$
E(r)=-\frac{m c^{2}}{q R \gamma}\left[\left(\frac{\varepsilon}{R}\right)^{2}\left(\frac{r^{3}}{R^{3}}\right)+\frac{2 I}{I_{c} \beta \gamma}\left(\frac{R}{r}\right) \operatorname{erf}\left(\frac{r^{2}}{R^{2}}\right)\right]
$$

\section{BEAM BUNCHING}

One-dimensional problem of beam bunching with space charge has an approximate analytical solution [6]. Consider non relativistic beam of energy $\mathrm{qU}_{\mathrm{o}}$ with fixed radius $\mathrm{R}$ propagating in a tube of radius a. Injected beam passes through the gap of length $d$ with applied voltage $\mathrm{U}(\mathrm{t})=\mathrm{U}_{1} \cdot \sin \omega_{\mathrm{O}} \mathrm{t}$. In drift space particles are bunched which is characterized by the value of first harmonic of induced current $I_{1}$ as a function of bunching parameter $X$

$$
\frac{I_{1}}{I}=2 J_{1}(X), \quad X=\left(\frac{U_{1} M_{1}}{2 U_{0}}\right)\left(\frac{\omega_{0} Z}{v}\right)\left[\frac{\sin \left(\frac{\omega_{\mathrm{q}} Z}{v}\right)}{\left(\frac{\omega_{\mathrm{q}} \mathrm{Z}}{\mathrm{v}}\right)}\right],
$$

where $J_{1}(X)$ is a Bessel function, $M_{1}$ is a coupling coefficient of the beam with modulation gap:

$$
\mathrm{M}_{1}=\frac{\sin (\theta / 2)}{\theta / 2},
$$

$\theta=\omega_{\mathrm{o}} \mathrm{d} / \mathrm{v}$ is a transit time angle trough the gap, $\omega_{\mathrm{q}}=\sqrt{\mathrm{F}} \omega_{\mathrm{p}}$ is a reduced plasma frequency of the beam, $\omega_{\mathrm{p}}=2(\mathrm{c} / \mathrm{R}) \sqrt{\mathrm{I} /\left(\beta \mathrm{I}_{\mathrm{c}}\right)}$ is a plasma frequency for an unbounded beam and $\mathrm{F}$ is a form factor of reduction of plasma frequency due to finite radius of the beam and tube:

$\mathrm{F}=2.56 \mathrm{~J}_{1}^{2}\left(2.4 \frac{\mathrm{R}}{\mathrm{a}}\right) /\left[1+5.76 / \theta_{\mathrm{R}}^{2}\right], \quad \theta_{\mathrm{R}}=\omega_{\mathrm{o}} \mathrm{R} / \mathrm{v}$

Numerical value of the first harmonic of bunched beam is calculated as follow:

$$
\frac{I_{1}(z)}{I}=\frac{2}{N} \sqrt{\left[\sum_{n=1}^{N} \cos \omega_{0} t_{n}(z)\right]^{2}+\left[\sum_{n=1}^{N} \sin \omega_{o} t_{n}(z)\right]^{2}},
$$

where $t_{n}(z) i=1, \ldots N$ is a time of reaching of the particle with number $n$ of point $\mathrm{z}$. Numerical example of bunching of $150 \mathrm{keV}, 1 \mathrm{~A}, \mathrm{R} / \mathrm{a}=0.8$ proton beam represented by $10^{4}$ particles on the $256 \times 256$ grid is given in Fig. 4 .

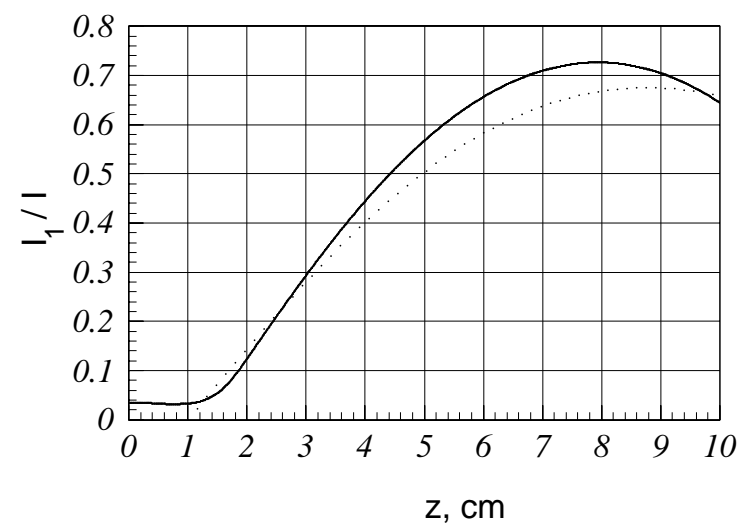

Fig. 4. Beam bunching: dotted line - analytical solution, Eq. (10); solid line - numerical values.

\section{REFERENCES}

[1]. I.M.Kapchinsky, Theory of Resonance Linear Accelerators, Harwood, Chur, 1986.

[2]. S.I.Molokovsky, A.D.Sushkov, Intense Electron and Ion Beams, Energoatomizdat, Moscow, 1991 (in Russian).

[3] D.L.Bruhwiler and Y.K.Batygin, Proceedings of the PAC95, Dallas, (1995) 3254

[4] Y. K.Batygin, Phys. Review E, 53, 5358, (1996).

[5] A.S.Chikhachev, Sov. Phys. Tech. Phys. 29 (9), 1984, p.990.

[6] Yu.A.Katsman, Microwave Devices, Moscow, 1973 (in Russian). 